\title{
Nordiques
}

38 | 2019

Le petit héros scandinave

\section{Créer sa propre histoire : Alternance des voix et discours métanarratif dans La vie commence de Stefan Casta}

\section{Annelie Jarl Ireman}

\section{(2) OpenEdition Journals}

Édition électronique

URL : http://journals.openedition.org/nordiques/292

DOI : $10.4000 /$ nordiques.292

ISSN : 2777-8479

Éditeur :

Association Norden, Bibliothèque de Caen la mer

\section{Édition imprimée}

Date de publication : 1 novembre 2019

Pagination : 59-71

ISBN : 979-1-0959140-4-4

ISSN : $1761-7677$

\section{Référence électronique}

Annelie Jarl Ireman, «Créer sa propre histoire : Alternance des voix et discours métanarratif dans La vie commence de Stefan Casta », Nordiques [En ligne], 38 | 2019, mis en ligne le 31 octobre 2020, consulté le 13 mars 2021. URL : http://journals.openedition.org/nordiques/292 ; DOI : https://doi.org/ $10.4000 /$ nordiques.292 


\section{Créer sa propre histoire : Alternance des voix et discours métanarratif dans La vie commence de Stefan Casta}

Annelie Jarl Ireman *

\section{RÉSUMÉ}

Cet article propose une analyse du discours narratif et métanarratif $d u$ roman $\mathrm{La}$ vie commence de Stefan Casta, qui permet de constater que l'auvre sinscrit dans une nouvelle tradition au sein de la littérature de jeunesse, caractérisée par sa forme expérimentale. L'alternance des voix, les changements de points de vue et de temps, ainsi que la structure influencée par le cinéma rendent le livre exigeant. Le narrateur principal ne fait pas que raconter son histoire, complétée par d'autres voix, il la transforme en roman. Grâce à cette création littéraire, Viktor évolue et trouve finalement le courage de commencer réellement sa vie. Son existence se confirme par le roman quil écrit, c'est en cela qu'il se montre hérö̈que. Le roman de Casta permet aux jeunes lecteurs de sidentifier eux-mêmes, notamment grâce au style accessible et aux références au cinéma, tandis que le discours narratif complexe attire des lecteurs plus expérimentés. C'est cette double-adresse qui définit avant tout la littérature de jeunesse suédoise d'aujourd'hui.

\section{ABSTRACT}

This article presents an analysis of the narrative and meta-narrative discourse of Stefan Casta's novel Näktergalens saing (The song of the nightingale), which shows that the book takes part in a new tradition in youth literature, characterized by an experimental form. The alternation of voices, changes of points of view and time, as well as the structure influenced by the cinema make the book demanding. The main narrator does not only tell his story, that other voices complete, he turns it into a novel. Thanks to this literary creation, Viktor evolves and finally finds the courage to really begin his life. His existence is confirmed by the novel he writes, and this makes him a hero. The novel by Casta allows young readers to identify themselves thanks to the accessible style and the references to cinema, while the complex narrative discourse attracts more experienced readers. It is this double-address which characterizes above all the Swedish youth literature of today.

* Annelie Jarl Ireman est maître de conférences au Département d'Etudes Nordiques à l'université de Caen Normandie. Attachée à l'ERLIS (Équipe de Recherche sur les Littératures, les Imaginaires et les Sociétés), elle est spécialisée dans le domaine de la littérature de jeunesse scandinave. 
Dans beaucoup de livres de jeunesse, il est impossible d'ignorer la présence du narrateur. C'est souvent le jeune protagoniste lui-même qui raconte son histoire. Certains romans pour adolescents scandinaves d'aujourd'hui sont très complexes, ce qui montre une évolution intéressante du genre. Comme le constate Ulla Lundqvist, cette évolution commence en Suède dans les années 1980 et concerne alors surtout les sentiments (les livres devenant moins "idylliques ») et la langue (syntaxe et vocabulaire plus complexes), mais également la forme littéraire qui devient plus expérimentale. ${ }^{1}$ A partir des années 1990, c'est avant tout dans la forme que nous pouvons constater un changement. Kajsa Bäckius, qui analyse l'œuvre de Per Nilsson, parle d'une nouvelle tradition qui se caractérise par la forme expérimentale pour ce qui est de la forme et de la structure, et par l'influence d'autres médias comme le cinéma. ${ }^{2}$ Un auteur représentatif à cet égard est Stefan Casta, lauréat notamment des Prix Alma (Astrid Lindgren Memorial Award), August (Augustpriset) et Nils Holgersson (Nils Holgersson-plaketten). Il a écrit une cinquantaine de livres pour enfants et adolescents, dont La vie commence, paru en Suède sous le titre Näktergalens sång ${ }^{3}$ en 2005 et traduit en français par Agneta Ségol en $2009^{4}$. Pour ce livre, l’auteur a été nominé pour le Prix August en 2005 et pour le Prix de littérature de jeunesse du Conseil nordique (Nordiska Barnbokspriset) en 2006. Nous allons voir que la modernité de ce livre réside avant tout dans un discours narratif complexe qui le rend exigeant et permet de le considérer comme de la littérature cross-over $^{5}$ et l'inscrit dans un univers post-moderne.

La vie commence parle de Victor qui a 19 ans, mais qui habite toujours chez ses parents adoptifs dans la campagne suédoise. Il ne sait pas vraiment qui il est ou ce qu'il veut faire dans la vie. De ce point de vue, c'est encore un enfant malgré son âge. Comme les jeunes restent en général plus longtemps chez leurs parents aujourd'hui, l'adolescence dure plus longtemps. Sa situation va changer avec l'arrivée d'une fille, un peu plus jeune que lui. Elle est censée être en famille d'accueil chez les voisins mais préfère rester chez eux où elle atterrit comme par hasard. Victor est le narrateur principal de l'histoire, qu'il crée en même temps. Quels sont donc ses objectifs et sa position par rapport à l'histoire?

1 Ulla Lundqvist, Tradition och förnyelse : svensk ungdomsbok frän sextiotal till nittiotal, Stockholm, Rabén \& Sjögren, 1994, p. 277-279.

2 Kajsa Bäckius, Per Nilsson som formmässig förnyare : en narratologisk studie av Hjärtans Fröjd och Anarki, Mémoire de Master sous la direction de Boel Westin, Département de littérature, université de Stockholm, 1999, 26 p. (dactylo.), p. 2-3.

3 Stefan Casta, Näktergalens sång, Bromma, Opal, 2005.

4 Stefan Casta, La vie commence, traduit du suédois par Agneta Ségol, Paris, Éditions Thierry Magnier, 2009.

5 Le terme de cross-over est utilisé pour parler des œuvres qui abolissent la frontière entre livres pour adultes et livres pour enfants. Ces dernières décennies, le terme définit avant tout les livres écrits pour un jeune lectorat mais qui attirent aussi des lecteurs adultes, et qui traverse donc la frontière dans ce sens uniquement. 
Comment crée-t-il son récit et quels sont ses limites ? Quelles autres voix portent la narration, et dans quels mouvements narratifs ? Nous terminerons par une réflexion sur l'héroïsme de ce personnage principal mais aussi sur les lecteurs et leur compréhension de ce type de livres.

\section{De L’obJeCTIVITÉ AU RÉCIT}

\section{Les temps et objectifs narratifs}

Le roman commence par une sorte de préface écrite au présent de narration à la première personne, s'adressant à un narrataire qui est le lecteur, avec pour objectif : parler d'une fille. Il se trouve devant l'ordinateur, le récit est donc clairement son intention, mais il a du mal car c'est difficile d'écrire. Quand il abandonne momentanément, les idées lui viennent et l'histoire peut commencer.

La première partie qui s'intitule "L'automne " (et qui sera suivie par l'hiver et le printemps) constitue un retour en arrière, et commence par la rencontre qui a tout changé. C'est le début de l'histoire qui, comme le narrateur le précise, va durer une année. La rencontre est décrite dans trois chapitres dont le premier et le troisième s'intitulent "Victor » et celui du milieu "Esmeralda ». Dans le premier, le narrateur utilise le présent pour donner son état d'esprit et expliquer qu'il ne regrette pas cette année. Puis il commence à raconter au passé : il s’agit donc d'une narration ultérieure selon les termes de Genette ${ }^{6}$. La fin du livre révèle qu'une année seulement s'écoule entre le début des évènements et la fin de la narration. Nous comprenons aussi qu'il écrit en partie son texte au fur et à mesure que l'histoire avance, sous forme de journal intime. Casta alterne les deux types de narration à la première personne, à savoir simultanée et rétrospective. ${ }^{7}$ Il y a à la fois un présent et un passé de narration. Après le premier chapitre écrit au passé, les événements ayant lieu dans le passé vont être relatés au présent. En outre, le temps de l'analepse finira par rattraper le moment de la narration. Cette synchronisation ${ }^{8}$ efface la frontière initialement donnée entre le passé et le présent.

Le narrateur donne d'abord à la première personne sa version des faits jusqu'au moment-même où il rencontre la fille (qu'il a failli tuer d'un coup de fusil). Il décrit ce qu'était sa propre vie à ce moment-là et analyse en même temps ce qui s'est passé. Il laisse ensuite la place à un narrateur hétérodiégétique9 (étranger à l'histoire) qui, à la troisième personne, donne la version d'Esmeralda, de son

6 Gérard Genette, Figures III, Paris, Éditions du Seuil, 1972, p. 229.

7 Termes empruntés à Maria Nikolajevea, Barnbokens byggklossar, Lund, Studentlitteratur, 2010, p. 152.

8 Ibid., p. 209-210.

9 Gérard Genette, op. cit., p. 252. 
point de vue à elle et en expliquant ce que le narrateur à la première personne ne peut pas savoir, puisque cela a lieu juste avant la rencontre. La narration fait un petit retour en arrière comme si on pressait le bouton rewind pour revoir le moment en question d'un autre point de vue. Les références au cinéma sont nombreuses, nous y reviendrons. La version d'Esmeralda est donnée au présent car nous plongeons maintenant dans l'histoire, l'analepse commence. Si ce chapitre donne principalement le point de vue de la fille, cela n'empêche pas le narrateur hétérodiégétique d'en adopter un différent, en se mettant dans la tête d'un autre personnage que la fille rencontre. Grâce à cela, nous avons un éclairage extérieur qui nous montre comment la fille est perçue par les autres personnages. Ces changements de perspective arrivent très vite dans le texte.

Dans le chapitre suivant, c'est comme si la caméra se déplaçait pour que nous voyions à nouveau le garçon en scène, exactement au même endroit où nous l'avions laissé à la fin du premier chapitre. Cette alternance de focalisation interne permet à l'auteur de raconter un même événement de deux points de vue différents. Nous retrouvons alors le narrateur qui parle à la première personne, désormais au présent, et qui se présente à la fille. Comme l'objectif de Victor est de parler d'Esmeralda, il semble être un narrateur homodiégétique ${ }^{10}$, mais cela devient plutôt son propre récit que celui de la fille. Il est donc en réalité un narrateur autodiégétique ${ }^{11}$, c'est-à-dire pas seulement un personnage mais le héros de sa propre histoire, même s'il reste discret en se cachant derrière la fille dans la narration.

\section{Le choix de texte}

Après cette préface qui donne l'objectif de la narration et les trois chapitres décrivant la rencontre qui a tout changé, l'histoire continue de façon linéaire au présent, par la voix de Victor. Les intitulés des chapitres sont dès lors construits de la même façon, avec la date et la température extérieure. On apprend en effet que Victor écrit un texte qui, au départ, est un journal météorologique comme on en écrit souvent dans la campagne suédoise pour garder une trace du travail à la ferme, pour suivre l'évolution des saisons et de la météo et pouvoir comparer d'une année à l'autre. Cela explique donc le titre des chapitres reprenant un point important d'un tel journal, à savoir la date et la température, et aussi pourquoi ses fichiers sur l'ordinateur s'intitulent "Histoires de Södra Visunda " ${ }^{12}$, le lieu étant essentiel. Tout cela implique d'écrire des faits concrets et d'être objectif. Or Victor décide qu'il peut prendre des libertés et écrire les pensées comme elles lui viennent à l'esprit, "qu'on a le droit de donner une forme personnelle à ce qu'on

10 Ibid., p. 252.

11 Ibid., p. 253.

12 Stefan Casta, op. cit., p. 73. 
écrit. Que ça peut même être un avantage. Ça devient moins artificiel. ${ }^{13}$ Cela le débloque et le texte qu'il écrit devient une sorte de journal intime ou plutôt de livre de pensées. Comme c'est la fille qui va surtout occuper ses pensées, ce sera un récit sur elle et leur relation, par conséquent aussi sur lui-même. Il hésite ainsi entre les deux types de récits : journal météo, objectif, ou journal intime, plus personnel. Les faits concrets insérés rendent l'histoire crédible. En tant que lecteurs, nous avons l'impression qu'il raconte ce qui s'est réellement passé. Les dates et la température de la journée en début de chapitre renforcent ce réalisme, mais elles permettent aussi au narrateur de se souvenir des événements et de construire son texte de façon chronologique. En relisant ses notes, il peut se rappeler d'autres événements qui se sont déroulés autour de telle ou telle date. Il n’est pas limité par ces dates, mais les utilise comme point de départ. Victor est donc, au début, un narrateur fiable puisqu'il raconte seulement ce qu'il peut savoir et analyse les événements. Il relate les dialogues en respectant la voix et le langage de chacun. Esmeralda par exemple emploie des mots vulgaires quand elle est en colère. C'est la version de Victor, mais il fait des efforts pour raconter avec honnêteté. Comme la fille est un mystère pour lui, nous, lecteurs, ne la connaissons pas bien non plus. Parfois il se corrige, avec du recul et dans le présent de narration : «J'ai compris plus tard que j'avais tort. $"{ }^{14}$ Il choisit cependant d'omettre certaines informations le concernant, notamment qu'il est adopté (ce que nous n'apprenons qu'à la page 232). A travers ce glissement ${ }^{15}$, nous comprenons qu'il n'est pas aussi fiable qu'il veut nous le faire croire. Il semble omettre cette information parce qu'elle n'est pas importante, mais il se trompe puisque le fait d'être adopté a une influence sur son incertitude identitaire.

En octobre, Esmeralda avoue savoir qu'il écrit, ce qui l'énerve car c'est censé être secret. A ce moment il ne pense pas écrire une histoire, "seulement des notes $[\ldots]$ sur différentes choses. Plutôt une sorte de journal intime ${ }^{16}$. Il n'a pas encore l'ambition et la confiance en lui pour appeler cela un récit.

\section{La création d'un récit}

Au cours de la deuxième partie du livre, au début de l'hiver, l'aspect métanarratif s'intensifie et la narration change. Le 20 décembre, les évènements bouleversants de la journée l'inspirent et les mots "viennent avec une facilité qu'[il] ne connaissai $[\mathrm{t}]$ pas. $»^{17} \mathrm{Il}$ veut la comprendre et aussi comprendre comment elle est

13 Ibid., p. 61.

14 Ibid., p. 188.

15 Maria Nikolajeva, op. cit., p. 176 et Kajsa Bäckius, op. cit., p. 11, p. 13. Nikolajeva traduit le terme anglais de "slant» qui vient de Seymour Chatman par "glidning", que nous traduisons par " glissement ".

16 Stefan Casta, op. cit., p. 93.

17 Ibid., p. 146. 
en relation avec lui-même et avec Brigitte et Gustavo, ses parents à lui. Il réalise que le texte

ne parle que de [leur] vie quotidienne, mais c'est comme si, tout d'un coup, cette vie décollait et qu'une sorte de souffle pénétrait dans la grisaille qui se mettait à respirer et à vivre. Et c'est [lui] ce souffle. ${ }^{18}$

Il tente maintenant d'imaginer à partir de ce qu'il sait, d'inventer pour elle un passé et un futur. Il réalise à ce moment-là qu'il est en train d'écrire : "Ça m’arrive alors comme une illumination : j'écris! Oui j'écris ! $"{ }^{19}$ Le journal météorologique ou le journal intime devient à ce moment un récit, ce qui implique une certaine liberté. Le sentiment de libération est de courte durée : "Je ne m'étais jamais rendu compte à quel point les mots pouvaient être carrés, froids et vides de sens $»^{20}$, note-t-il quelques soirs plus tard, analysant ainsi ce que c'est d'écrire et constatant que pour « réellement décrire quelqu'un avec des images justes et vivantes, il faut trier les mots, il faut les tourner et les retourner jusqu'à ce que les bons apparaissent $»^{21}$. Il compare avec la peinture qui lui semble plus facile à utiliser pour parler d'elle, mais comme il n'a pas le matériel nécessaire, il continue à se battre sur l'ordinateur. Il réalise qu'il n'a qu' " une langue de tous les jours »", des mots pour parler de faits simples. Il croit que cela ne suffit pas, mais il va montrer qu'il est possible d'utiliser sa propre langue pour écrire.

A Noël, l'harmonie qui s'installe l'inspire à nouveau et il écrit une dizaine de pages presque sans s'en rendre compte. Esmeralda qui l'observe fait remarquer qu'il est "dans [s] on texte $»^{23}$. Il comprend que c'est vrai et se demande si, par conséquent, elle se trouve aussi dans le texte : "Et si c'était moi qui... la créais ! L'idée me donne le vertige. $»^{24}$ Existe-t-elle parce qu'il en parle dans son texte ? Ou est-ce que le texte existe parce qu'elle est là ? Le narrateur met ici en doute la véracité de l'histoire. Parle-t-il de ce qui se passe réellement ou est-ce de la pure fiction? Les deux se mêlent. Le narrateur lui-même ne le sait plus. L'auteur instaure ainsi un sentiment d'incertitude chez le lecteur, qui doit tenter d'interpréter les indices laissés dans le texte.

La fille encourage Victor dans l'écriture en lui donnant une définition qui correspond à sa propre vision :

Je crois que la réalité est un mythe, qu'elle est une sorte de récit que nous inventons au fur et à mesure. Nos vies sont des histoires qui se déroulent de

18 Ibid., p. 146-147.

19 Ibid., p. 147.

20 Ibid., p. 167.

21 Ibid.

22 Ibid., p. 171.

23 Ibid., p. 185.

24 Ibid. 
façon permanente et c'est nous qui leur donnons l'orientation. Ton histoire et la mienne se sont maintenant croisées et, ensemble, elles donnent naissance à une nouvelle historie : la nôtre. [...] Toi tu écris des textes qui arrivent à trouver leurs propres chemins. Tu crées de nouveaux mondes. ${ }^{25}$

Elle confirme à Victor, et par là même au lecteur, que le texte qu'il écrit est cette nouvelle histoire dont elle parle, qu'il a donc le droit de l'inventer. Quand il veut en savoir davantage sur elle pour pouvoir en parler, elle refuse car c'est lui " le narrateur $»^{26}$. Le problème, c'est que Victor a l'impression de perdre le contrôle, de ne plus savoir où se trouve la frontière entre la vérité et la fiction. Il finit par accepter que la fiction fait partie de la réalité : "Toute vie est une histoire racontée. ${ }^{27}$ Si le narrateur semble juste décrire ce qu'il sait au départ, un changement s'opère, il se met à fabuler, tout en restant crédible. Cela a pu se passer comme ça.

Il finit par laisser Esmeralda lire ce qu'il a écrit, utilisant le terme de "manuscrit $»^{28}$. Il assume alors le fait d'écrire un roman, dans le but d'être lu par d'autres. En effet, Victor a envie de devenir écrivain. Son texte est un ballon d'essai, pour se prouver qu'il en est capable. La seule personne qui soit au courant croit en lui. La fin du livre confirmera son talent et son avenir : être écrivain.

\section{Les vecteurs de l'imagination}

Un jour, il est de nouveau dans l'incapacité d'écrire ce qui s'est passé puisqu'il n'était pas avec la fille à un certain moment. Soudain il entend de la musique. Celle-ci déclenche une scène et sa capacité à imaginer ce qu'il ne sait pas : comme la musique de fond d'un film. Elle lui permet de voir la scène, c'est-à-dire de l'inventer :

[La musique] est partout à la fois, elle emplit la maison. Il me semble alors voir la fille, tout au fond de moi. Elle sort de la salle de bains, vêtue d'une chemise de nuit bleue avec des lapins blancs, elle va dans le salon, elle prend le manuscrit sur le canapé et s'installe dans le fauteuil en cuir à côté de la table. [...] Oui je vois très clairement un film passer dans ma tête. Un film où la fille joue l'unique rôle et où la musique du piano constitue un fond sonore permanent. $^{29}$

Cette technique de mise en scène l'aide dans l'écriture. Il voit le déroulement comme dans un film, crée un scénario, ce qui lui permet de visualiser ce qui se passe dans une autre pièce, pour ensuite le décrire avec des mots. En utilisant l'expression "dans ma tête ", il avoue ne pas le savoir mais l'imaginer. La technique n'est pas infaillible. Son imaginaire ne suffit pas et le film dans sa tête s'arrête en

25 Ibid., p. 193-194.

26 Ibid., p. 195.

27 Ibid., p. 196.

28 Ibid., p. 200.

29 Ibid., p. 200-201. 
même temps que la musique : "Ça bloque ici! Je n’y arrive plus. Et après ? Je ne vois plus la fille et je m’aperçois que la musique a cessé. » $^{30}$

Les références au monde cinématographique sont nombreuses. Le narrateur peint des scènes comme s'il était en train de filmer avec une caméra : nous l'avons constaté au début, avec les deux perspectives différentes. Quand la maison est encerclée par la police, Victor constate que c'est "comme au cinéma " ${ }^{31}$. Dès qu'il se passe quelque chose de dramatique comme une confrontation entre les personnages, le narrateur a recours à cette technique :

La réplique de Brigitte rompt le sortilège qui avait paralysé la cour. Nous reprenons soudain vie et nous nous remettons à bouger. Comme si une réalisatrice avec déambulateur avait dit : Action !32

Des termes comme "réplique ", "Action " ou encore "slow-motion " ${ }^{33}$ renforcent l'impression de scénario. Victor doit voir le film dans sa tête et entendre la musique, vecteurs de son imagination, pour pouvoir écrire. Or la technique cinématographique s'inscrit aussi dans la structure du texte, par des ellipses narratives, souvent implicites. Différentes scènes sont comme coupées et mises bout à bout. Dans un même moment de la narration, l'histoire avance en accéléré et le lecteur peut supposer que le temps est passé. ${ }^{34}$ Les dates indiquées nous aident alors à suivre la progression temporelle. Parfois, le narrateur fait comme un arrêt sur image, un étirement narratif ${ }^{35}$, ce qui veut dire que le temps de la narration est plus long que le déroulement de la scène, quand celle-ci est importante et dramatique. C'est le cas du passage qui se termine par la citation en exergue ci-dessus. C'est un moment clé de l'histoire puisque la fille refuse violemment de quitter la ferme pour aller dans sa famille d'accueil. Pour l'éviter, elle menace avec un fusil et finit par tirer sur la voiture du voisin. La détonation met les protagonistes dans un état de choque, le temps s'arrête et il faut deux pages et demie à Victor pour analyser ce qui se passe réellement en un instant. Cela lui donne le temps de commenter la scène, d'interpréter ce que ressentent les autres et ce qu'ils pensent. Par cette technique, l'auteur bloque le lecteur dans un moment dramatique et le libère quand il laisse Brigitte dire une phrase banale ramenant les personnages dans le quotidien : "Maintenant je veux mon cafi. " Le narrateur constate alors que l'action reprend, comme sur l'ordre d'un réalisateur.

30 Ibid., p. 202.

31 Ibid., p. 289.

32 Ibid., p. 88.

33 Ibid., p. 159.

34 Cf Kajsa Bäckius, op. cit., p. 20.

35 Maria Nikolajevea, op. cit., p. 222. Nous traduisons le terme «stretch" par étirement.

36 Stefan Casta, op. cit., p. 88. 


\section{LES VOIX ET VOIES DU RÉCIT}

\section{Les limites du narrateur principal}

Au milieu de la deuxième partie intitulée "Hiver ", la narration doit à nouveau changer parce que la fille s'en est allée sans explication. Victor ne peut alors plus savoir, ni imaginer ce qui se passe car elle est trop loin. Il laisse la place à un narrateur hétérodiégétique comme au début du livre, ce qui permet au lecteur de voir les événements du point de vue de la fille. On peut donc conclure que le déroulement n'est pas ce que Victor imagine mais fictionnellement vrai. Le chapitre porte le titre "Esmeralda Alice Caroline Louise " (à comparer avec "Esmeralda » au début du livre) car on y apprend son " véritable " prénom (qui s’avérera être celui de sa mère, selon la postface de l'auteur). Le chapitre suivant s'intitule "Victor" et c'est lui-même qui raconte à la première personne. Son prénom utilisé comme titre indique que cela se passe au même moment que les événements décrits dans le chapitre sur Esmeralda. Comme elle n'est pas directement dans son histoire à ce moment-là, le récit commun se divise en deux trames parallèles, ce qu'atteste le changement de titre et de narration. Quatre chapitres consacrés à la fille, en alternance avec quatre chapitres sur Victor, sont nécessaires avant que les deux histoires se rejoignent de nouveau et que le récit commun puisse continuer, ce qui est signalé par un retour aux dates et températures comme intitulés des chapitres. Quand la fille revient, Victor constate qu'elle a essayé de sortir de son histoire à lui "pour agir toute seule ${ }^{37}$. Or elle n'a pas ce pouvoir, puisque c'est son histoire à lui. Elle doit revenir pour qu'il puisse terminer à sa façon. Dans le récit, elle se trompe donc étrangement de chemin et finit par être ramenée par la factrice.

Dans un de ces textes sur Victor, celui-ci donne la parole à Brigitte, sa mère, pour qu'elle raconte sous forme de monologue ce qui s'est passé il y a longtemps ${ }^{38}$ Elle s'adresse à la dame des services sociaux mais donne en même temps les infirmations manquantes à Victor. Un peu plus tard, dans la dernière partie du livre intitulé "Printemps ", il passe la parole à la fille, pour qu'elle donne enfin son véritable prénom (Nora) et explique qui elle est. Si elle accepte finalement de le faire, c'est parce qu'elle préfère alors donner sa version et éviter qu'ils ne l'apprennent par l'assistante sociale ou en lisant son dossier. Le lecteur est enfin sur le point d'avoir des informations fiables sur la fille, puisque celles-ci viennent directement d'elle. Or, juste avant qu'elle ne commence son récit, la musique se met à jouer de nouveau. ${ }^{39}$ L'histoire qui suit est assez invraisemblable. Nora est en réalité la petite fille de Brigitte et donc la nièce (par adoption) de Victor. Son père à elle, un grand pianiste, a été renversé en traversant la rue à Vienne, tandis

37 Stefan Casta, $o p$. cit., p. 233.

38 Ibid., p. 229-232.

39 Ibid., p. 294. 
que sa mère, actrice, a fini par se suicider. Cette mère était la fille que Brigitte a eue dans sa jeunesse et qu'elle a confiée à l'adoption, longtemps avant d'adopter Victor. La musique qui signalait auparavant que Victor commençait à imaginer, nous laisse à penser que cela se passe peut-être dans sa tête et que la fille raconte quelque chose de plus banal. Victor constate d'ailleurs ne pas avoir "entendu " son histoire, mais " l'avoir vue », comme " un film à l'intérieur de [lui] ». ${ }^{40}$ L'histoire de la fille ne contredit pas celle de Brigitte mais, comme le constate Victor lui-même, elle semble construite :

Je ne suis pas absolument certain de prendre son histoire pour argent comptant. Ça me parait un peu trop bien. Ce sont des choses qui n'arrivent que dans les livres ou dans les films. Quand elle essaie elle-même d'inventer sa vie, ça manque de naturel, à mon avis, ça fait un peu artificiel. ${ }^{41}$

Il comprend cependant que Brigitte a envie d'y croire et qu'il est sans importance que ce soit vrai ou non puisque chaque vie est un récit que nous écrivons nousmêmes. Nous avons donc parfaitement le droit de l'inventer.

\section{Les textes réels-fictionnels}

Comme on l'a vu, les notes de Victor sur son ordinateur sont insérées dans l'histoire pour la soutenir. D'autres textes apparaissent dans le livre, comme par exemple la lettre d'un éditeur, grâce auxquels l'histoire se complète. Tout en étant fictifs, ces documents accentuent l'aspect réaliste du roman. Le livre se termine ainsi par une lettre de Nora à Victor, ce qui permet une adresse au narrateur à la deuxième personne, exactement un an après le début de l'histoire, et qui confirme que Victor a alors terminé la rédaction de son roman. Avec cette lettre, nous pouvons enfin entendre directement la voix de la fille, sans le risque d'une déformation de la part du narrateur principal. Cette lettre montre que Nora aussi a douté de l'histoire, puisque les écrivains " mentent, c'est leur boulot. Bizarrement, il leur arrive de mentir avec une telle conviction que ça devient presque de la réalité. $»^{42}$ Elle justifie ainsi le doute du lecteur sur ce que Victor a raconté. Elle apporte pourtant une preuve par la suite, en expliquant qu'elle avait découvert des coupures de presse dans les papiers de sa mère après son suicide. Grâce à ces articles, elle a pu découvrir la vérité. Nous comprenons qu'elle n'était pas venue par hasard, mais dans le but précis de retrouver sa grand-mère. Victor a imaginé avec une telle conviction que cela s'accorde avec la réalité. L'auteur du livre apporte lui-même une dernière preuve quand il prend la parole en son propre nom dans une sorte de postface, dans laquelle il constate que ses recherches

40 Ibid., p. 297.

41 Ibid., p. 298-299.

42 Ibid., p. 325. 
confirment le résultat de Victor. Mais un auteur a le droit de mentir, le livre nous l'a appris, ce texte n'est bien entendu pas plus fiable que les autres.

\section{La naissance d'un jeune homme}

Grâce à l'écriture, Victor se construit, grandit. Au début de l'histoire, il se sent complètement perdu. Il pense manquer de modèles adultes et ne pas être à sa place, " comme un jeune coucou qui grandit dans un nid qui n'est pas le sien. ${ }^{43}$ Son état d'esprit se retrouve dans le texte. Au début, il associe librement, les pensées vont et viennent dans sa tête. Le lecteur a parfois du mal à suivre. Or cela s'améliore grâce à l'écriture. Pendant longtemps, nous ne savons pas qui il est, ni pourquoi il est là, puisque ce n'est pas, a priori, une histoire sur lui mais sur elle. Il se décrit également comme un "valet de ferme $»^{44}$. Nous pouvons imaginer qu'il est placé dans une famille d'accueil, et le comportement de la mère le confirme quand l'assistante sociale vient en visite. Plus tard, on apprend pourtant qu'il a été adopté au Sri Lanka quand il était bébé : il est donc le fils des personnes qu'il appelle par leurs prénoms. S'il cache ainsi des informations importantes sur luimême, c'est parce qu'il tente de garder une distance en parlant de la fille, mais aussi parce qu'il est à la recherche d'une identité propre et ne s'assume pas encore. L'arrivée d'Esmeralda est un facteur déclencheur, poussant les trois membres de la famille à changer, à s'ouvrir. Esmeralda ouvre un chemin vers autre chose pour Victor, une possibilité de changement radical. Cette ouverture est d'abord symbolisée par le modem qu'il achète, une fenêtre vers le monde, vers les études, et un autre avenir.

Le mouvement narratif suit les saisons et correspond à l'évolution des personnages. L'histoire se termine ainsi logiquement par une renaissance au printemps, pour Victor mais aussi pour la fille et pour la mère. Le récit de Victor va en effet petit à petit quitter Nora, pour se rapprocher de Kenny, un jeune homme rencontré à l'université et de qui Victor semble tomber amoureux. Kenny est celui qui le pousse finalement à partir et commencer réellement sa vie.

À présent, nos histoires se séparent. Un jeune coucou s'envole, un autre s'installe. D'autres histoires pourront commencer. ${ }^{45}$

C'est le moment où sa vie de jeune adulte débute, comme le titre l'indique, ainsi que son prénom. C'est celui qui réussit à prendre son envol et à se trouver, sans l'aide des adultes. Son héroïsme consiste, comme dans de nombreux livres pour adolescents, à avoir trouvé le courage de s'assumer et de grandir, même s'il n'est qu'au début de cette évolution identitaire. Or Victor a également réussi à transformer sa vie en récit. Le jour du déménagement, il découvre dans la voiture

\footnotetext{
43 Ibid., p. 79.

44 Ibid., p. 159.

45 Ibid., p. 323.
} 
une enveloppe contenant la réponse d'un éditeur. Nora s'était en effet absentée pour envoyer une partie de son manuscrit pour avis. L'éditeur souhaite lire la suite, ce qui confirme la qualité de son récit.

En racontant, ce narrateur autodiégétique s'adresse régulièrement aux lecteurs, pour leur rappeler que ce sont eux les narrataires du texte, qu'ils doivent rester concentrés et retenir les faits, ou pour donner des explications. Il montre aussi qu'il fait confiance aux lecteurs, qu'il n'a pas besoin de se répéter. ${ }^{46}$ Le lecteur est donc incité à lire de façon active et entrer en relation avec le narrateur. Si le récit a permis à Victor lui-même de se construire, ce en quoi réside son hérö̈sme, les lecteurs peuvent rester perplexes quant à la vérité fictionnelle de l'histoire. L'alternance des voix, parfois contradictoires, et le discours métanarratif complexe créent ensemble cette incertitude, typique de la littérature postmoderne. Le lecteur doit juger la vérité fictionnelle de l'histoire, rester attentif aux changements de voix et de perspectives, décider qui croire et compléter l'histoire avec ce que les différents narrateurs peu fiables ne peuvent ou ne veulent pas dire.

Le nouveau jeune héros scandinave serait-il alors le lecteur? Les jeunes lecteurs suédois habitués à ce genre de livres auraient-ils développé les capacités nécessaires pour les comprendre ? On peut en effet se demander s'ils arrivent à faire tout ce que l'auteur exige de leur part, ou si ce genre de narration est trop complexe pour quelqu'un ayant peu d'expérience de la lecture. Daniel Delbrassine constate en parlant du roman pour adolescents suédois que cet emploi de "structures narratives complexes suppose un lecteur déjà initié aux formes littéraires contemporaines $"{ }^{47}$ Des études de réception concernant d'autres livres tout aussi exigeants montrent que les filles apprécient davantage ces défis que les garçons, qui sont souvent moins habitués à lire. ${ }^{48}$ Nous pouvons supposer que certains éléments du discours narratif et la métafiction risquent de passer inaperçus aux yeux de certains jeunes lecteurs, mais ceux-ci ressentent sans doute l'ambiguïté voulue. Les lecteurs plus expérimentés apprécieront peut-être aussi davantage la réflexion sur l'écriture. En revanche, le style, avec des marqueurs d'oralité et un langage parfois familier, permet aux adolescents de s'identifier et de lire sans difficulté, ce

46 Quelques exemples : "Je suppose que vous l'avez compris. " (159) ; "Vous commencez à les connaitre maintenant, n'est-ce pas? » (170) ; "Si vous connaissez le sourire de la Joconde, vous voyez la différence. » (185) ; «- Waouf! Répond... qui vous savez. » (235)

47 Daniel Delbrassine, Le roman pour adolescents aujourd'hui : écriture, thématiques et réception, Paris, SCEREN - CRDP de l'académie de Créteil / La Joie par les livres, 2006, p. 68.

48 Voir Helen Schmidt : Från vildmark till grön ängel : Receptionsanalyser av läsning $i$ àttonde klass, Makadam, 2008, p. 111-159. (L'étude concerne Hjärtans fröjd de Per Nilsson (Rabén \& Sjögren, 1992), traduit en français par Agneta Ségol sous le titre Faux raccord (Thierry Magnier, 2008.) 
que la traductrice a réussi à garder en français, en partie par compensation..$^{49}$ Les jeunes lecteurs apprécient sans doute particulièrement les références au cinéma. Il devient pour eux plus facile d'entrer dans l'histoire en la voyant comme un film. Enfin, la métafiction semble être un élément clé de la littérature de jeunesse d'aujourd'hui et les jeunes lecteurs peuvent sans doute facilement s'identifier avec ce besoin de raconter sa vie, que ce soit par le biais des blogues et des réseaux sociaux ou par des moyens plus traditionnels. L'existence même se confirme par la création du récit. Le grand nombre de visites effectuées par l'auteur dans les écoles, ainsi que les nombreuses critiques écrites par des adolescents sur Internet montrent en tout cas que ses livres sont lus et appréciés des jeunes.

L'auteur fait preuve d'un grand respect envers son lecteur en comptant sur ses capacités à saisir le texte, c'est " un livre d'adulte pour jeunes ", comme l'a exprimé un critique littéraire dans un journal. ${ }^{50}$ Ceci constitue à mon sens la particularité de la littérature de jeunesse scandinave de ces dernières décennies, qui est ici représentée par Stefan Casta. Ce genre a formellement (et d'ailleurs aussi du point de vue thématique) rompu la frontière qui le séparait de la littérature pour adultes. Ainsi, les méthodes d'analyse traditionnellement utilisées pour les livres destinés aux adultes, peuvent aussi être appliquées dans ce type de livres, qui ont également leurs particularités en tant que littérature de jeunesse. ${ }^{51} \mathrm{Si}$ le roman de Casta s'adresse principalement aux adolescents ${ }^{52}$, il s'agit d'une littérature pour tous, novatrice et de grande qualité littéraire. Grâce à la double-adresse, le genre s'est ouvert vers davantage de lecteurs sans pour autant désintéresser les jeunes. C'est la littérature cross-over par excellence.

49 Charlotte Lindgren, "He speaks as children speaks : more orality in translations of modern Swedish children's books into French ? ", in Martin B. Fischer, Maria Wirf Naro (réd.), Translating fictional dialogue for children and young people, Berlin, Frank \& Timme, 2012, p. 180.

50 « en vuxenbok för unga » Expressen, 5/12/2005. Notre traduction.

51 Cf. Maria Nikolajeva, op. cit., p. 13-48 et Kajsa Bäckius, op. cit. p. 23-24.

52 L'éditeur Opal indique "à partir de 14 ans ». Site de l'éditeur Opal disponible sur : http://www. opal.se/index.php?p=pres\&isbn=9789172991712\#.W4UrHegzaUk (consulté le 27/08/2018). 